\title{
Optical Design of a Solar Dish Concentrator Based on Triangular Membrane Facets
}

\author{
Hongcai Ma, ${ }^{1,2}$ Guang Jin, ${ }^{1}$ Xing Zhong, ${ }^{1}$ Kai Xu, ${ }^{1}$ and Yanjie $\mathrm{Li}^{1,2}$ \\ ${ }^{1}$ Changchun Institute of Optics, Fine Mechanics and Physics, Chinese Academy of Sciences, Changchun 130033, China \\ ${ }^{2}$ Graduate University of the Chinese Academy of Sciences, Beijing 100039, China \\ Correspondence should be addressed to Guang Jin, jing@ciomp.ac.cn
}

Received 30 June 2012; Revised 13 October 2012; Accepted 17 October 2012

Academic Editor: Kalvala Srinivas Reddy

Copyright ( $) 2012$ Hongcai Ma et al. This is an open access article distributed under the Creative Commons Attribution License, which permits unrestricted use, distribution, and reproduction in any medium, provided the original work is properly cited.

\begin{abstract}
The design of a solar dish concentrator is proposed based on triangular membrane facets for space power applications. The facet concentrator approximates a parabolic surface supported by a deployable perimeter truss structure, which originates from a large aperture space antenna. For optimizing the number of facets rows and focal-diameter ratio of the concentrator, Monte Carlo ray-tracing method is utilized to determine optical performance of the concentrator, and the system root-mean-square (RMS) deviation is considered in this design procedure. A 600 -facet concentrator with focal-diameter ratio of 1.1 will achieve $83.63 \%$ of radiative collection efficiency over a $15 \mathrm{~cm}$ radius disk located in the focal plane, with a mean solar concentration ratio exceeding 300. The study in this paper is helpful for the development of the membrane facet concentrator.
\end{abstract}

\section{Introduction}

Space-based solar energy is an attractive energy source with a huge potential application for the space solar power and support of various spacecrafts. Researchers have focused on researching the large aperture concentrator as the key component for space-based concentrator photovoltaic (CPV) system collecting space-based solar radiation onto a highefficiency photovoltaic cells $[1,2]$. To reduce volume and mass of space-based CPV systems while maximizing efficiency of the photovoltaic cells, there has been long interest in the development of membrane dish concentrators for space power applications. As lightweight, inexpensive, highperformance structures, they are excellent candidates for space-deployable collectors as well as cost-effective terrestrial energy sources. For the terrestrial use, the proposed design of ellipsoidal polyester membrane facets has much better performances than a multifacet dish concentrator consisting of identical circular, and the simulated membrane shape was experimentally verified [3]. A large-span solar parabolic trough concentrator is designed based on a multilayer polymer mirror membrane mounted on a rotatable concrete structure [4]. For the use in space, an inflatable prototype for the Deployable Solar Concentrator has been developed [5]. Especially, L'Garde has developed power antenna concept, and this new technology utilizes an inflatable membrane reflector to concurrently concentrate solar energy for space electrical power generation, while acting as an antenna with large aperture and high gain [6]. Similarly, many reflector antennas with a mesh membrane surface and a deployable perimeter truss, such as AstroMesh antenna [7, 8] and deployable modular mesh antenna [9], divide the reflector surface into triangular flat facets. While the above antenna configurations are also adaptive to be used as concentrators, they had not been explored for solar power generation applications.

This paper deals with the optical design of a lightweight solar concentrator with triangular membrane flat facets as potential low-cost alternate for space solar power generation applications. The focal radiation flux distributions are calculated by the Monte Carlo ray-tracing method. To obtain the desired radiative collection efficiency and mean solar concentration ratio, we investigate on the minimal number of facets of the concentrator when varying focal-diameter ratio $(F / D)$ of the concentrator under consideration of both the system RMS deviation and the random surface error. 


\section{Concentrator Geometry}

Generally, the deployable membrane antennas consist of cable network supplemented with a membrane, tension ties, perimeter truss, and rear net. For simplicity, we only consider the forming process for a membrane surface. The number and size of triangle facets of the membrane surface are determined by layout of nodes. To minimize the number of nodes, a net with coinciding three-band nodes is adopted in this paper [10]. The layout of nodes is shown as Figure 1(a). For the circular aperture, the membrane is divided into $m$ equal sectors and $N$ equally spaced concentric circles, and there are $m \times n$ nodes in the $n$th concentric circles lying on equally spaced radian length of $2 \pi /(m n)$. Then the horizontal nodes are mapped onto the desired parabolic surface giving the shape of membrane after deformation. In reality, the horizontal nodes of membrane are pulled down by cable to the mapped positions of nodes, and then the nearby three nodes comprise a triangle membrane facet. The mapping procedure used for generating the triangular-facet membrane surface is shown in Figure 1(b). The ideal deformed membrane is approximately parabolic in shape and consists of many triangular flat planes, but in reality each plane becomes deformed due to cable tension, membrane tension, membrane size, and surface curvature which is related to focal length. The ideal forming process for a membrane surface has only been considered in this paper. In the final surface, the number of triangular facets $t$ and nodes $j$ are

$$
\begin{gathered}
t=m N^{2}, \\
j=m N^{2}+1,
\end{gathered}
$$

and numerical description of the deformed membrane given by the positions of nodes on the parabolic surface is derived as

$$
P_{\text {nodes }}=\left(\begin{array}{l}
x_{n, i} \\
y_{n, i} \\
z_{n, i}
\end{array}\right)=\left(\begin{array}{c}
n \frac{D}{2 N} \cos \left[(i-1) \frac{2 \pi}{m n}\right] \\
n \frac{D}{2 N} \sin \left[(i-1) \frac{2 \pi}{m n}\right] \\
\frac{\left(x_{n, i}+y_{n, i}\right)}{4 F}
\end{array}\right) \quad 1 \leq i \leq m n
$$

where $n$ is the number of concentric circles where the nodes are located, $i$ represents the $i$ th node in the anticlockwise direction in the $n$th concentric circle, $D$ is the aperture diameter, and $F$ is the nominal focal length of the concentrator.

For the solar concentrator based on a triangular membrane facets, in this paper we assume the requirements for the radiation flux on a circular target are that radiative collection efficiency $\eta_{c}=80 \%$, at a high mean concentration ratio of at least $C_{\text {mean }}=300$; meanwhile, the concentrator geometry is refined by selecting the minimum number of facets rows $N$, and the $F / D$ ratio is varied to get the desired radiation flux as high as possible.

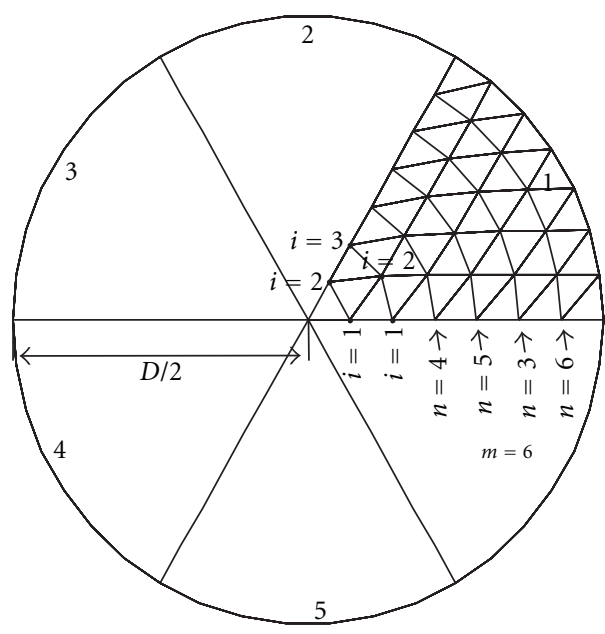

(a)

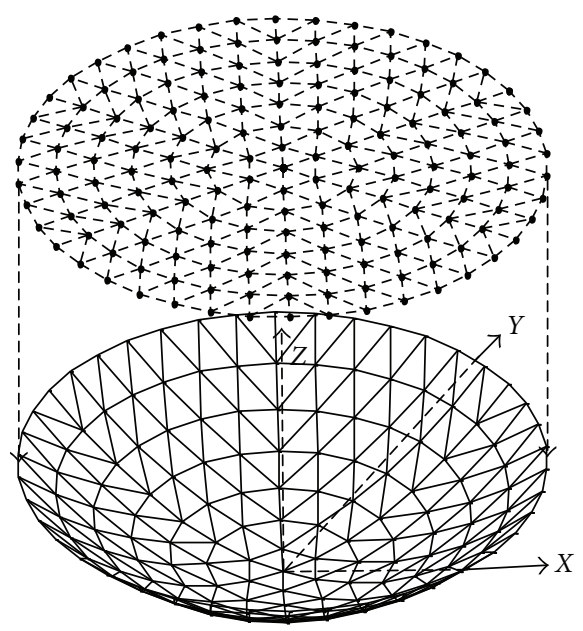

(b)

FIGURE 1: Flat-facet concentrator: (a) a layout of nodes with $m=6$ and $t=294$ facets, placed in $N=7$ rows and (b) the mapping procedure for generating the triangular facets membrane surface.

\section{Monte Carlo Ray-Tracing Method}

In Monte Carlo ray-tracing method, the average radiation flux on the target $\bar{q}$ and the area-weighted mean solar concentration $C_{\text {mean }}$ on a circular disk target are defined as

$$
\begin{gathered}
\bar{q}=\frac{\pi \sum_{m=1}^{N_{\text {target }}} q_{m}\left(r_{m}^{2}-r_{m-1}^{2}\right)}{\pi R_{\text {target }}^{2}}, \\
C_{\text {mean }}=\frac{\bar{q}}{I_{0}},
\end{gathered}
$$

where $q_{m}$ is the radiation flux received by the $m$ th discrete annular element, $r_{m}$ is the radius of the $m$ th discrete annular element, $N_{\text {target }}$ is the number of discrete annular elements on the circular target, $R_{\text {target }}=0.15 \mathrm{~m}$ is the radius of the circular target disk, and $I_{0}$ is the incident direct solar flux in space.

Incident rays are distributed over the triangular elements in proportion to the projected element areas. The angular distribution of the launched rays is modeled by 
TABle 1: Parameters of the simulated concentrator.

\begin{tabular}{ll}
\hline Parameters & Numerical value \\
\hline Concentrator aperture & $D=6 \mathrm{~m}$ \\
Focal-diameter ratio & $F / D=0.4 \sim 1.6$ \\
Incident direct solar flux & $I_{0}=1309.2 \mathrm{~W} / \mathrm{m}^{2}$ \\
Target size & $R_{\text {target }}=0.15 \mathrm{~m}$ \\
Receiver type & Flat, without shadowing \\
Number of facets & $N=9,10,11,12$ \\
Half-cone angle of sun disk & $\theta_{\text {sun }}=4.65 \mathrm{mrad}$ \\
Surface error & $\sigma_{s}=0,10 \mathrm{mrad}$ \\
Reflectivity of facets & 1 \\
equal sectors divided & $m=6$ \\
\hline
\end{tabular}

the "pillbox-sunshape", which represents the ideal solar disk with uniform brightness in half-cone angle $\theta_{\text {sun. }}$. In order to take into account the random surface error combining with membrane wrinkling at the facets, a Gaussian distribution (expressed in milliradians) is assumed for the angular deviation of the reflected rays with respect to the nominal direction of reflection [11]. The latter is given by the specular law of reflection in which triangular-facets normal vectors are derived from the node triples positions. The receiver is considered without shadowing the incident solar energy. Table 1 summarizes the parameters of the membrane facet concentrator.

\section{Optical Design of Triangular-Facets Concentrator for the System}

The accuracy requirements of deployable antenna with a perimeter truss structure are often specified by the system RMS deviation $\delta_{\text {rms }}$ of the actual triangular flat facets surface from the desired parabolic surface. While we could vary the $F / D$ ratio of the concentrator originated from the deployable antenna to get the focal radiation flux as high as possible, the allowable RMS deviation limits the satisfying range of $F / D$ ratios. Thus, the RMS deviation is considered for this analysis. For a given value of $\delta_{\text {rms }}$, the maximal triangular facet length $L$ and the facet rows $N$ are given by [12]

$$
\begin{gathered}
\frac{L}{D}=\sqrt{8 \sqrt{15} \frac{\delta_{\mathrm{rms}}}{D} \frac{F}{D}}, \\
N=\frac{D}{2 L} .
\end{gathered}
$$

According to the practical limitation of the allowable RMS deviation $\delta_{\text {rms }}=0.36 \mathrm{~mm}$ [13], the relationships between the facet rows $N$ and the minimum $F / D$ ratio are shown by the curve in Figure 2. In Figure 2, the $F / D$ ratios satisfying the requirement of the RMS deviation are on the upper right zone of the curve, and there are satisfying $F / D$ ratios for $N=9,10,11$, and 12 , respectively. The satisfying range of $F / D$ ratios enlarges when the facet rows $N$ increases. With the concentrator divided into an enough large number of small facets, all range of $F / D$ ratios is satisfying. This relationship offers restricted limits for the $F / D$ ratio selected

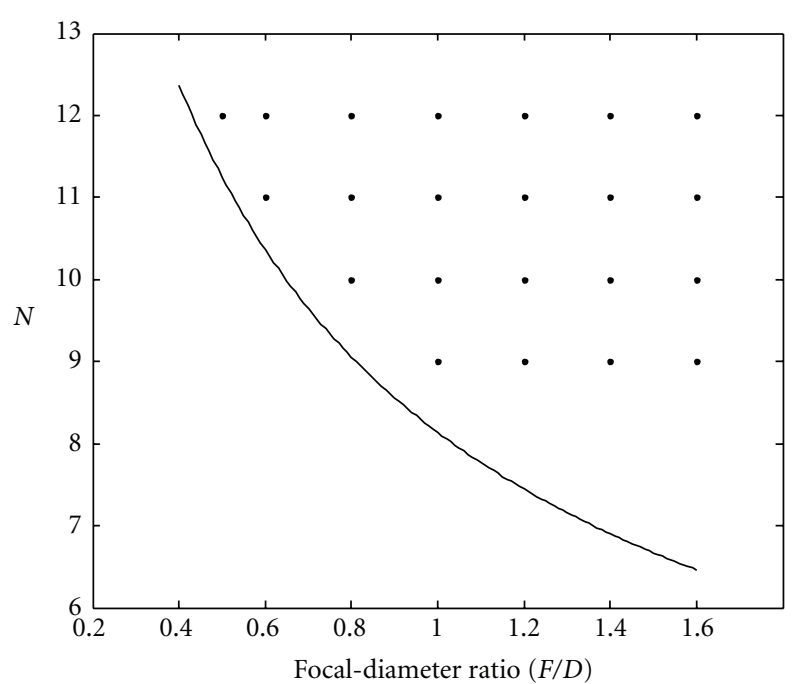

FIGURE 2: The dependence of the number of facet rows $N$ on minimum $F / D$ ratio with the allowable RMS deviation $\delta_{\text {rms }}=0.36 \mathrm{~mm}$.

for different facet rows $N$ in the following optimization procedure of the flat-facet concentrator.

Figures 3(a) and 3(b) show that the variation of radiative collection efficiency with $F / D$ ratio when the random surface error $\sigma_{s}$ is 0 and $10 \mathrm{mrad}$, respectively, for the facet rows $N$ of $9,10,11$, and 12 . Both figures illustrate that the larger the number of facets, the higher the collection efficiency. By comparing (a) and (b), it is shown that the collection efficiency $\eta_{c}$ is slightly lower in the presence of random surface error for the same facet rows $N$ value. For instance, by comparison of the case with and without the surface error, the average decreasing amount is only $0.1 \%$ from 0.4 to 1.6 of $F / D$ ratio. In addition, an optimal $F / D$ ratio which corresponds to the highest collection efficiency exists for each facet rows $N$ as the horizontal line shows; however, the optimal $F / D$ ratio decreases with the increase of facet rows $N$. For instance, for a concentrator with $N=10$, the collection efficiency $\eta_{c}$ increases from $67.7 \%$ to $85.27 \%$ for the $F / D$ ratio increasing from 0.4 to 1.1 and with $N$ increasing from 9 to 12 , the optimal $F / D$ ratio decreases from 1.3 to 1.1 . Furthermore with the $F / D$ increasing beyond the optimal $F / D$ ratio, the collection efficiency decreases more obviously with random surface error than without random surface error, for example the average decrease is $0.3 \%$ with surface error of $10 \mathrm{mrad}$ and $0.15 \%$ without random surface error when $F / D$ ratio increases from 1.1 to 1.6 for $N=10$.

Similarly, Figures 4(a) and 4(b) show the variation of the mean concentration ratio $C_{\text {mean }}$ with the $F / D$ ratio for several facet rows $N$ values when the random surface error $\sigma_{s}$ is 0 and 10 mrad, respectively. The mean concentration ratio $C_{\text {mean }}$ exhibits similar dependence on $F / D$ ratio as the collection efficiency does (Figure 3 ). The mean concentration ratio also increases with the increasing number of facet rows $N$. With the surface error increasing from 0 to $10 \mathrm{mrad}$, the mean concentration ratio $C_{\text {mean }}$ appreciably decreases. When $N=$ $10, F / D=1.1$, the mean concentration ratios are 340.9, 333.5 , respectively, both exceeding 300 . 


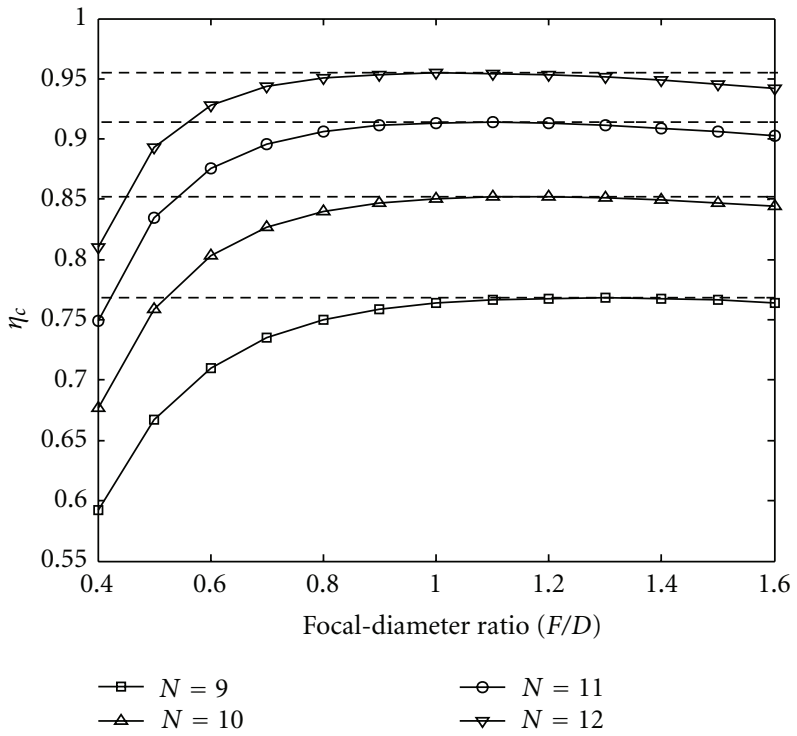

(a)

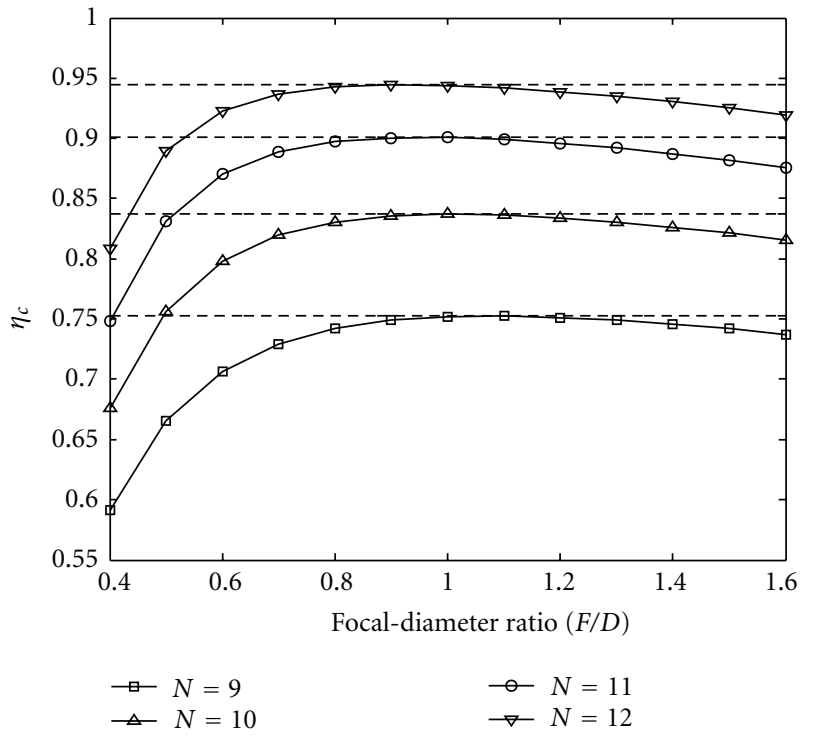

(b)

FIgURE 3: The dependence of radiative collection efficiency $\eta_{c}$ on $F / D$ ratio with the random surface error $\sigma_{s}$ of (a) $0 \mathrm{mrad}$ and (b) $10 \mathrm{mrad}$.

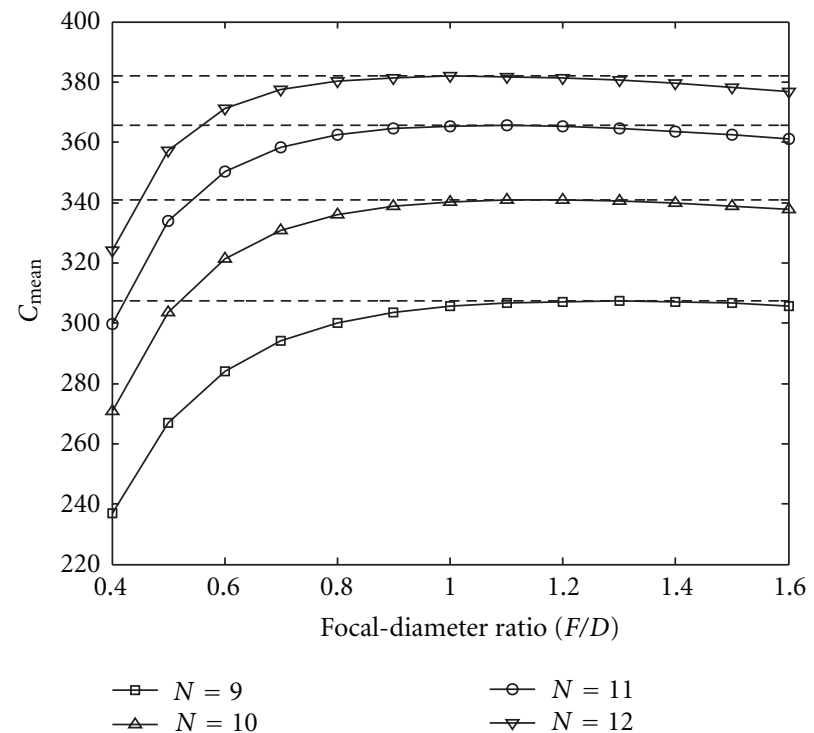

(a)

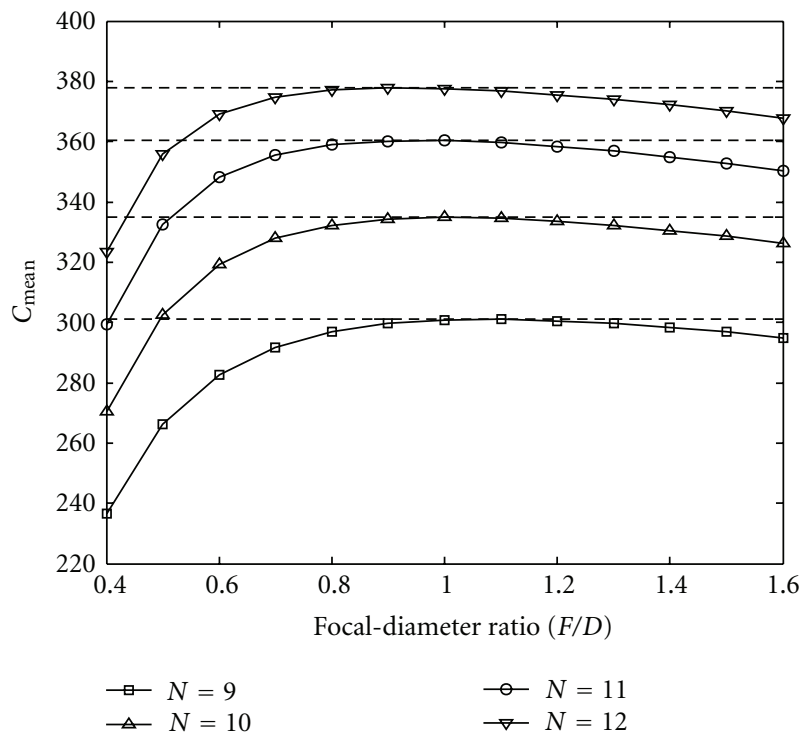

(b)

FIGURE 4: The dependence of the area-weighted mean solar concentration $C_{\text {mean }}$ on $F / D$ ratio with the random surface error $\sigma_{s}$ of (a) 0 mrad and (b) 10 mrad.

In the case keeping concentrator aperture $D$ constant, the existence of the optimal $F / D$ ratio for each facet rows is discussed in more detail. When the $F / D$ ratio increases, the concentrator implies a reflected solar cone with more angular divergence. Therefore, the image on the receiver increases. But, on the other hand, a smaller $F / D$ ratio also implies larger RMS deviation, which increases the image size. Therefore, the observed optimal $F / D$ ratio is the result of a trade-off between these two effects.

Based on the above analysis, the selection of the optimal facet rows $N$ and $F / D$ ratio is determined by both the desired flux and allowable RMS deviation $\delta_{\text {rms }}$. The procedure of optimization design can be described in the following steps: firstly, the minimum number of facet rows $N$ is obtained according to the requirements of radiation flux; secondly, the appropriate range of $F / D$ ratio is derived from the allowable RMS deviation $\delta_{\text {rms }}$; finally, the $F / D$ ratio is scanned over the range satisfying the requirement of the RMS deviation to achieve the highest radiation flux under the consideration of the random surface error. Based on this design procedure, the obtained optimal facet rows $N=10$ and $F / D$ ratio $=1.1$ result in radiative collection efficiency of $83.63 \%$ at a mean 
concentration ratio of 333.5 for a $15 \mathrm{~cm}$ radius target with the surface error of $10 \mathrm{mrad}$, which fulfills well the design requirements.

\section{Conclusions}

We have identified the optical performance of a solar concentrator based on triangular membrane facets for energy collection. The concentrator approximates an ideal parabolic with triangular flat facets supported by a deployable perimeter truss structure, which originates from a large aperture space antenna. An optical design procedure to the membrane facet concentrator has been developed. The number of facet rows $N$ and focal-diameter ratio $(F / D)$ was optimized by Monte Carlo ray-tracing method, in which the radiative collection efficiency and area-weighted mean concentration ratio were calculated. Under consideration of the allowable RMS deviation $\delta_{\text {rms }}=0.36 \mathrm{~mm}$ and a random surface error combing with membrane wrinkling of $10 \mathrm{mrad}$, a 600 facet concentrator will achieve $83.63 \%$ of radiative collection efficiency over a $15 \mathrm{~cm}$ radius disk located in the focal plane, with an area-weighted mean concentration ratio of 333.5. The solar concentrator based on triangular membrane facets is a promising option for the development of a cost-effective space photovoltaic solar energy generation.

\section{Acknowledgment}

The financial support provided by National Basic Research Program of China with Grant no. 2010CB227101 is greatly appreciated.

\section{References}

[1] M. J. O’Neill, M. F. Piszczor, M. I. Eskenazi et al., "Ultralight stretched Fresnel lens solar concentrator for space power applications," in Proceedings of the SPIE's 48th Annual Meeting, pp. 5179-5117, San Diego, Calif, USA, August 2003.

[2] L. S. Mason, "A solar dynamic power option for space solar power," NASA/TM 1999-209380, 2000.

[3] G. Zanganeh, R. Bader, A. Pedretti et al., "A solar dish concentrator based on ellipsoidal polyester membrane facets," Solar Energy, vol. 86, pp. 40-47, 2012.

[4] R. Bader, A. Pedretti, and A. Steinfeld, "A 9-m-aperture solar parabolic trough concentrator based on a multilayer polymer mirror membrane mounted on a concrete structure," Journal of Solar Energy Engineering, vol. 133, no. 3, Article ID 031016, 2011.

[5] G. Grossman and G. Williams, "Inflatable concentrators for solar propulsion and Dynamic Space Power," Journal of Solar Energy Engineering, vol. 112, no. 4, pp. 229-236, 1990.

[6] D. Lichodziejewski and C. Cassapakis, "Inflatable power antenna technology," in Proceedings of the AIAA 99-1074 37th Aerospace Sciences Meeting and Exhibit (AIAA '99), January 1999.

[7] K. Ando, J. Mitsugi, and Y. Senbokuya, "Analyses of cablemembrane structure combined with deployable truss," Computers and Structures, vol. 74, no. 1, pp. 21-39, 2000.

[8] N. M. Awlad Hossain, C. H. Jenkins, and L. Hill, "Analysis of a membrane modified perimeter-truss mesh antenna," in
Proceedings of the UV/Optical/IR Space Telescopes: Innovative Technologies and Concepts, pp. 259-270, SPIE, Bellingham, Wash, USA, August 2004.

[9] S. P. Chodimella, J. Moore, J. Otto, and H. Fang, "Design evaluation of a large aperture deployable antenna," in Proceedings of the AIAA/ASME/ASCE/AHS/ASC Structures, Structural Dynamics, and Materials Conference, vol. 6624, Newport, RI, USA, May 2006.

[10] G. Tibert, Deployable tensegrity structures for space applications [Ph.D. thesis], Stockholm, 2002.

[11] A. Rabl, Active Solar Collectors and Their Applications, Oxford University Press, New York, NY, USA, 1985.

[12] P. K. Agrawal, M. S. Anderson, and M. F. card, "Preliminary design of large reflectors with flat facets," IEEE Transactions on Antennas and Propagation, vol. AP-29, no. 4, pp. 688-694, 1981.

[13] M. W. Thomson, "AstroMesh deployable reflectors for ku and ka band commercial satellites," in Proceedings of the 20th International Communication Satellite Systems Conference and Exhibit (AIAA '02), Montreal, Canada, May 2002. 


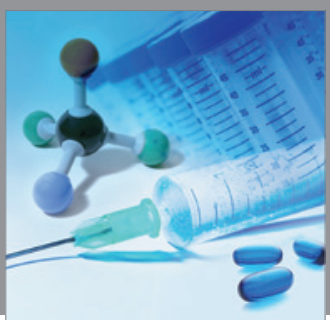

International Journal of

Medicinal Chemistry

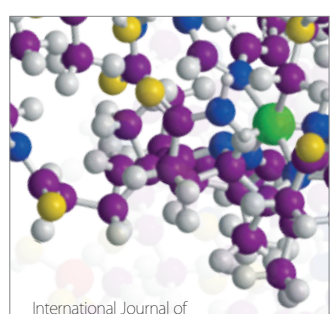

Carbohydrate Chemistry

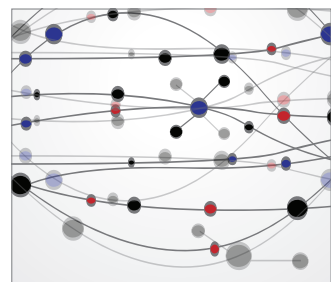

The Scientific World Journal
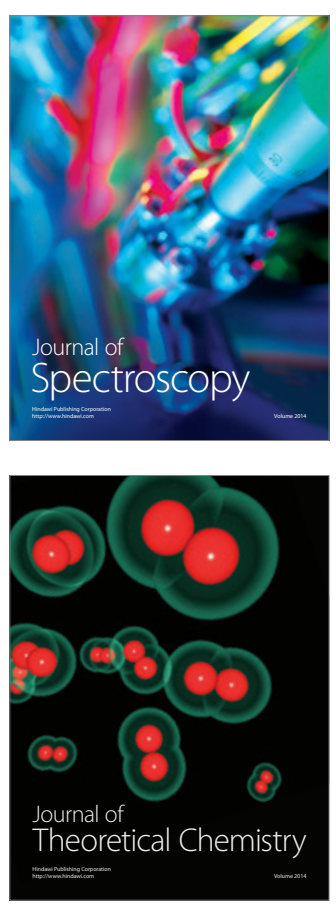
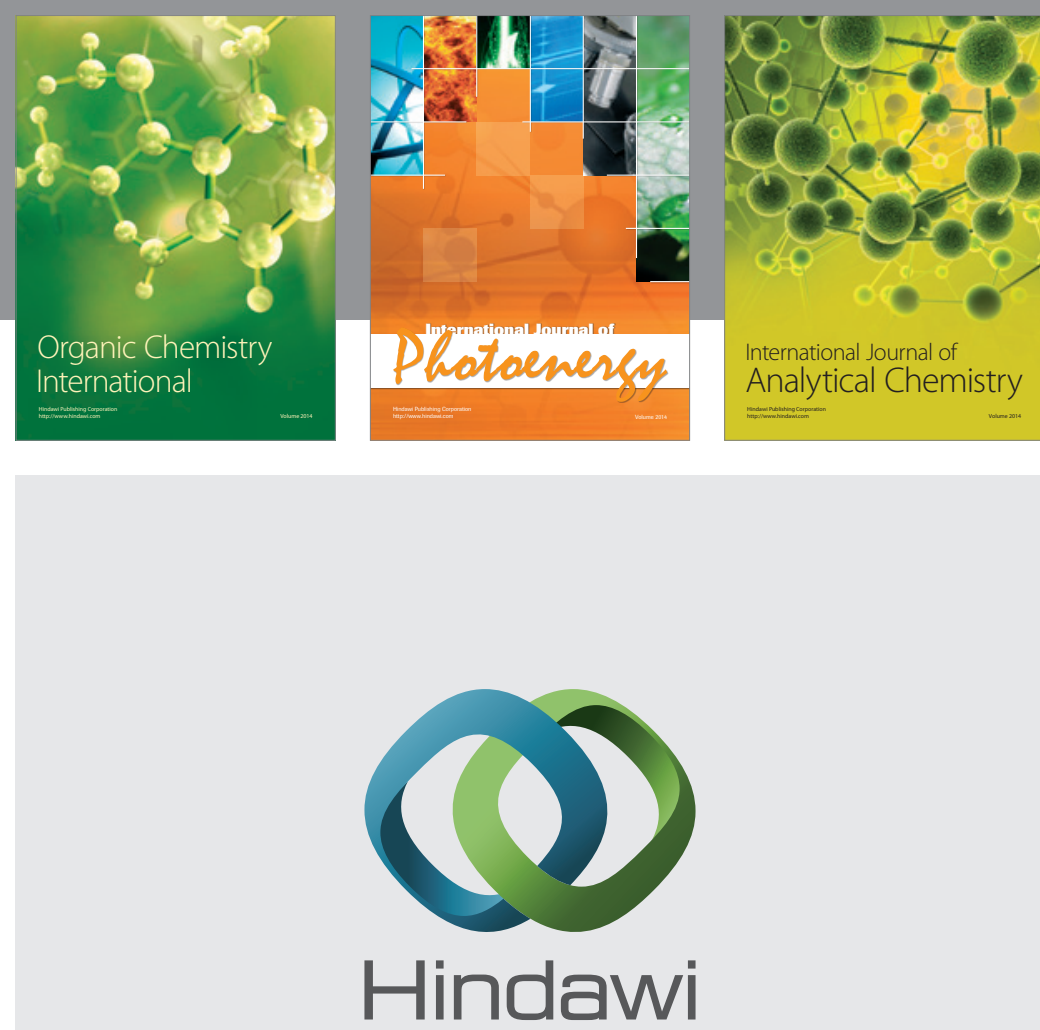

Submit your manuscripts at

http://www.hindawi.com
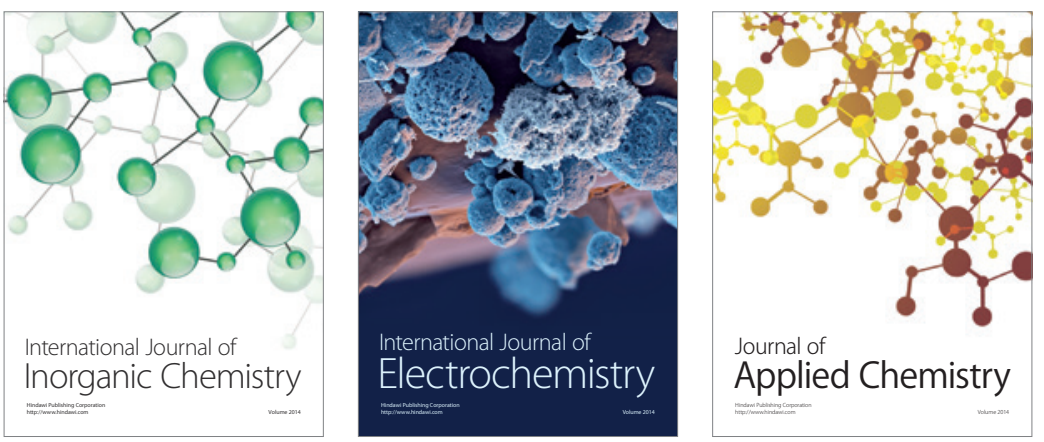

Journal of

Applied Chemistry
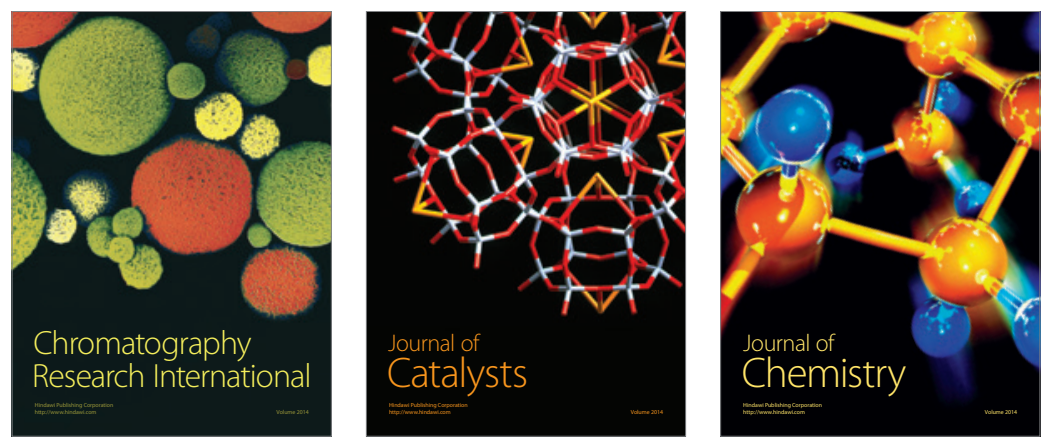
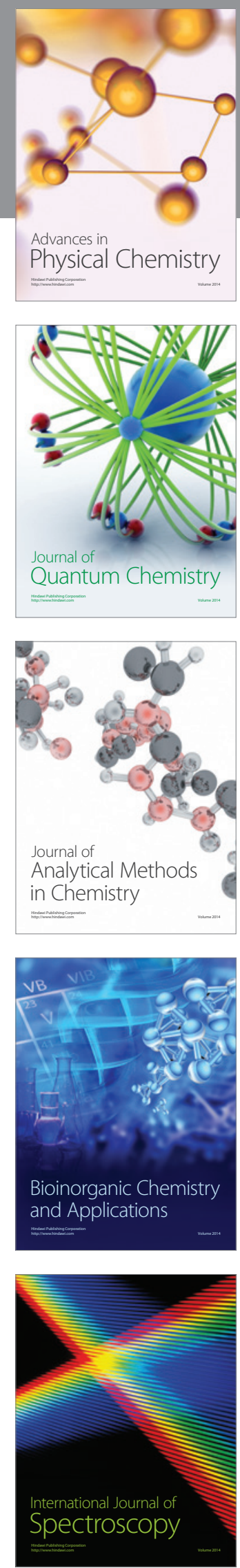